Case Report

\title{
Spontaneous Intracranial Hypotension Treated with a Targeted CT-Guided Epidural Blood Patch
}

\author{
Inês Correia, ${ }^{1}$ Inês Brás Marques, ${ }^{1}$ Rogério Ferreira, ${ }^{2}$ Miguel Cordeiro, ${ }^{3}$ and Lívia Sousa ${ }^{1}$ \\ ${ }^{1}$ Department of Neurology, Centro Hospitalar e Universitário de Coimbra, Coimbra, Portugal \\ ${ }^{2}$ Department of Internal Medicine, Centro Hospitalar e Universitário de Coimbra, Coimbra, Portugal \\ ${ }^{3}$ Department of Neuroradiology, Centro Hospitalar e Universitário de Coimbra, Coimbra, Portugal \\ Correspondence should be addressed to Inês Correia; mcorreia.ines@gmail.com
}

Received 20 November 2015; Revised 13 January 2016; Accepted 14 January 2016

Academic Editor: Masahiro Kohzuki

Copyright (C) 2016 Inês Correia et al. This is an open access article distributed under the Creative Commons Attribution License, which permits unrestricted use, distribution, and reproduction in any medium, provided the original work is properly cited.

\begin{abstract}
Spontaneous intracranial hypotension (SIH) is an important cause of new daily persistent headache. It is thought to be due to spontaneous spinal cerebrospinal fluid (CSF) leaks, which probably have a multifactorial etiology. The classic manifestation of $\mathrm{SIH}$ is an orthostatic headache, but other neurological symptoms may be present. An epidural blood patch is thought to be the most effective treatment, but a blind infusion may be ineffective. We describe the case of a young man who developed an acute severe headache, with pain worsening when assuming an upright posture and relief gained with recumbency. No history of previous headache, recent cranial or cervical trauma, or invasive procedures was reported. Magnetic resonance imaging showed pachymeningeal enhancement and other features consistent with SIH and pointed towards a cervical CSF leak site. After failure of conservative treatment, a targeted computer tomography-guided EBP was performed, with complete recovery.
\end{abstract}

\section{Introduction}

Spontaneous intracranial hypotension $(\mathrm{SIH})$ is a known cause of new daily persistent headache. Although the classic feature is an orthostatic headache, other symptoms related to the pathophysiology of SIH, such as neck pain or stiffness, nausea, vomiting, imbalance, or visual complaints, may also be present with variable clinical severity. Conservative treatment is usually attempted first; however, this approach is unsuccessful in many patients. In these cases, an epidural blood patch (EBP) is usually considered. It is common to perform a blind EBP, but it can be ineffective and several trials may be necessary to achieve success, with some patients being refractory to this approach. A targeted CT-guided EBP is a more recent treatment option, with a higher success rate reported, but technical expertise is needed.

\section{Case Report}

We report the case of a 34-year-old man who presented to our emergency department complaining of a severe, diffuse, and throbbing headache. The headache had started two weeks before and shown a progressive worsening with a gradual increase in intensity during this period. The pain was immediately exacerbated when the patient assumed an upright position but was partially relieved after approximately five minutes of recumbency. Common analgesics brought no relief. The patient also complained of nausea, vomiting, photophobia, the sensation of neck stiffness from headache onset, and episodic double vision during the previous two days. He denied having a history of previous headache, recent head or neck trauma, or invasive procedures. There was a personal medical history of psoriasis and asthma, but he was asymptomatic at the time. He attended the gym on a daily basis and he had played tennis on the day before the headache onset. On the fourth day of symptoms, the patient resorted to going to the emergency department of a local hospital, where a brain computerized tomography (CT) was requested. The exam did not show any abnormal findings, and the patient was discharged with symptomatic analgesics. 


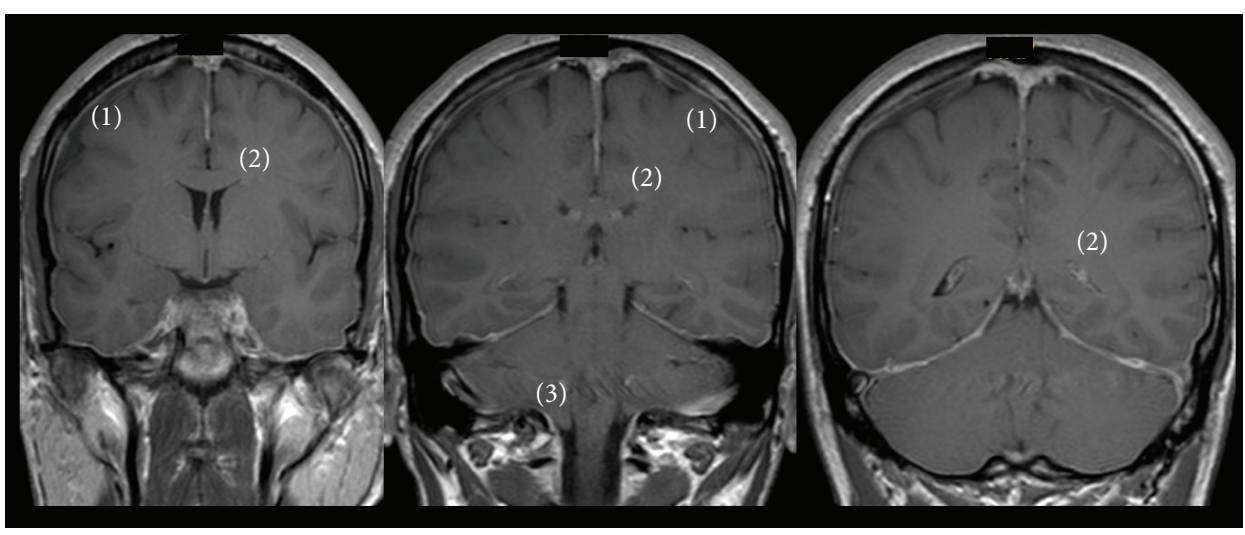

(a)

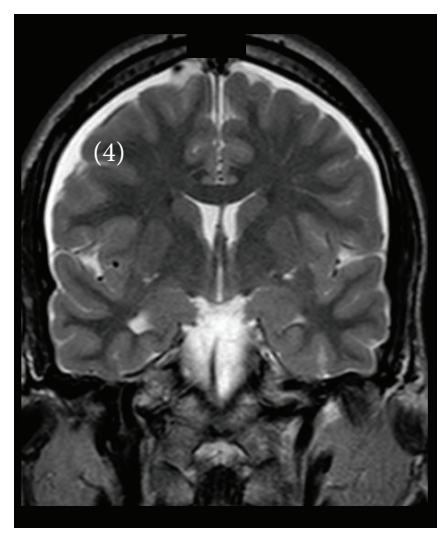

(b)

FIGURE 1: (a) T1-weighted brain MRI scan with gadolinium showing diffuse pachymeningeal enhancement (1), decrease in ventricle size (2), and cerebellar tonsil descent (3). (b) T2-weighted brain MRI scan showing subdural fluid collections (4).

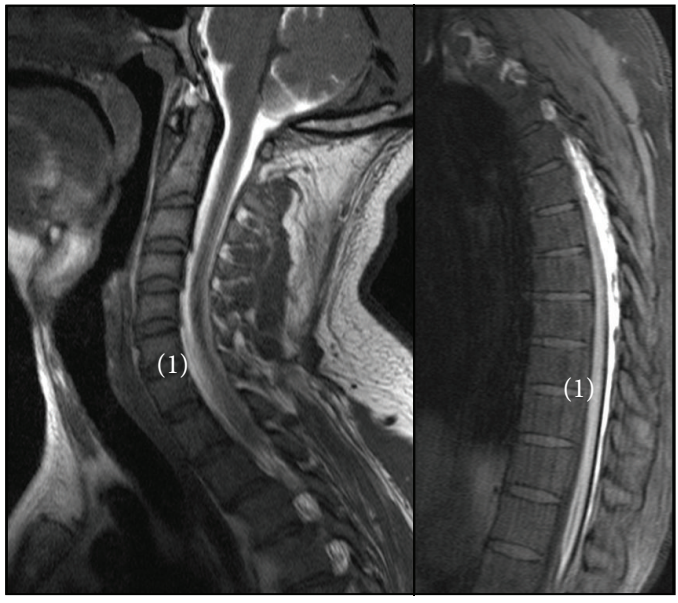

(a)

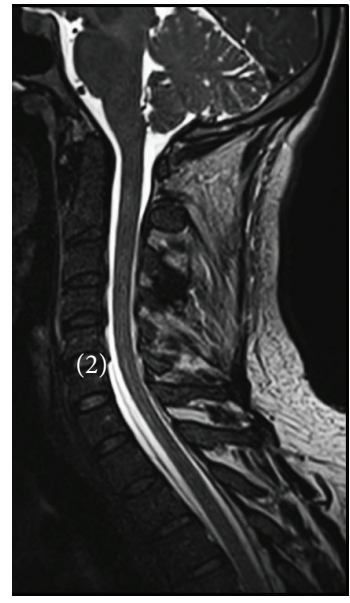

(b)

FIGURE 2: (a) T1-weighted spine MRI scan after intrathecal administration of gadolinium: sagittal views showing extra-arachnoid cerebrospinal fluid collections enhanced with gadolinium (1). (b) T2-weighted spine MRI scan showing subdural fluid collections and site of leak (2).

The physical and neurological examination on admission to our emergency department were remarkable only for a left sixth nerve paresis and severe photophobia.

A brain magnetic resonance imaging (MRI) scan was performed and revealed diffuse pachymeningeal enhancement, a decrease in ventricle size, cerebellar tonsil descent, and subdural fluid collections, findings which were compatible with SIH (Figure 1).

MRI myelography was then requested. A low cerebrospinal fluid (CSF) opening pressure $\left(5 \mathrm{~cm} \mathrm{H}_{2} \mathrm{O}\right)$ was confirmed during the procedure and a spread of CSF in the epidural space was observed, extending from L3 to the cervical region, entering the intrathecal space at $\mathrm{C}$, suggesting a CSF leak located at this level (Figure 2).

A conservative approach was initially tried, consisting of bed rest, hydration, analgesia, and caffeine intake. After two weeks without clinical improvement, it was decided to proceed with an EBP. Since the CSF leak was located at a cervical level and the literature suggests that these cases are less likely to resolve with a blind EBP, we chose to perform a targeted CT-guided EBP. It involved a CT-guided injection of $10 \mathrm{~mL}$ of autologous blood with $2.5 \mathrm{~mL}$ of iodinated contrast targeted at the D2-D3 epidural space, with a satisfactory blood spread into the epidural space between $\mathrm{Cl}$ and D6 (Figure 3).

On the day after the targeted EBP was carried out, there was a pattern change in the headache, with a worsening in the supine position, which was suggestive of intracranial hypertension. An urgent CT-scan did not show any new findings, indomethacin was prescribed, and the patient was asymptomatic after three days of treatment. Brain MRI performed one month later revealed a complete resolution of the imagiological features and the patient remained without headache recurrence after 30 months. 


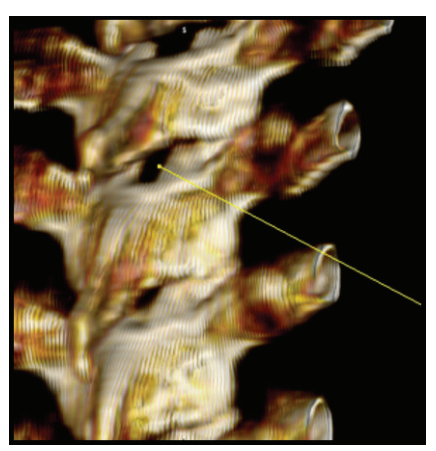

(a)

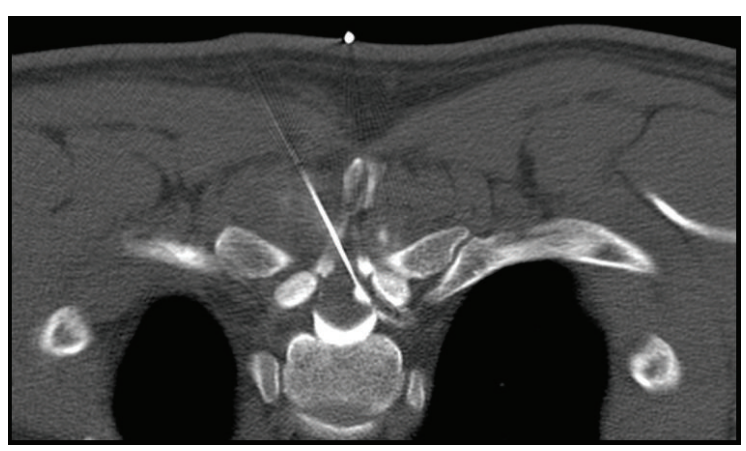

(b)

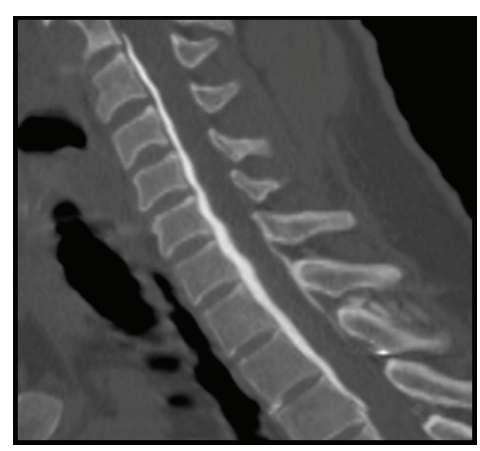

(c)

Figure 3: (a) Spine CT-scan three-dimensional simulation site for targeted EPB. (b) Targeted CT-guided EBP. (c) Spine CT-scan showing contrasted blood injected in EBP.

\section{Discussion}

SIH is increasingly being recognized as a cause of new daily persistent headache with an estimated annual incidence of $5 / 100,000$ and an incidence peak of around 40 years of age $[1,2]$.

It is due to spontaneous spinal CSF leaks from a tear in the dural membrane, which occurs most often at the reflection of the pia mater to dura mater at the root exit zones, mostly in the cervical region or cervicothoracic junction, although other possible sites are the thoracic, lumbar, and even sacral regions and occasionally the vestibular system, the cribriform plate, or the pituitary fossa [3, 4]. However, a definite location of the leak is not precisely established in a significant number of cases [4]. In a case series of 56 patients with SIH the locations of CSF leakage were cervical in 6 patients, cervicothoracic in 15 , mid-lower thoracic in 8 , lumbar in 10, and unknown in 17 [5].

Spontaneous spinal CSF leaks probably have a multifactorial etiology, with patients with underlying disorders of the connective tissue having an increased risk of SIH and some cases being preceded by minor trauma, such as coughing, falling, or sports activities [1]. A wide variety of dural defects identified during surgery have been described, such as simple dural holes, meningeal diverticula, and absence of the dura normally covering the spinal nerve root [6].

The classic manifestation of SIH is an orthostatic headache that usually starts within seconds to minutes after assuming an upright position and is relieved following recumbency. A wide range of other neurological symptoms have been described in SIH. More than half of the patients present with posterior neck pain or stiffness, nausea, or vomiting. Between $10 \%$ and $50 \%$ of patients complain of changes in hearing and a disturbed sense of balance. Less than $10 \%$ have visual blurring, visual field defects, diplopia, facial numbness, facial pain, facial weakness, facial spasm, dysgeusia, parkinsonism, or ataxia. Cerebellar haemorrhage and posterior fossa subdural hygroma, dementia, interscapular pain, quadriplegia, upper extremity radicular symptoms, hyperprolactinemia, and galactorrhea have also been described [1, 2, 6-8].
The clinical features of our patient, orthostatic headache accompanied by nausea, vomiting, photophobia, binocular diplopia, and neck stiffness, were very suggestive of this condition.

Brain MRI is important in the evaluation of patients when $\mathrm{SIH}$ is suspected. Diffuse pachymeningeal enhancement, a decrease in ventricles size, cerebellar tonsil descent, and subdural fluid collections are findings consistent with $\mathrm{SIH}$, as described in our patient. Spinal MRI may show extraarachnoid CSF collection, meningeal diverticula, pachymeningeal enhancement, epidural venous plexus engorgement, and bone abnormalities, which may have contributed to the dural tear. Imagiological techniques such as CT myelography or MRI myelography may be used to confirm the diagnosis and to document the site of the leak. Nuclear cisternography and myelography have also been used in the past to confirm the diagnosis $[1,2,7]$.

The initial recommended approach includes bed rest, analgesia, caffeine administration, and hydration, although there is no class A or B evidence for this approach $[1,2]$. When this conservative treatment fails, an EBP is considered the mainstay of treatment for SIH $[1,4,7]$. The proposed mechanisms of action for an EBP include the formation of a dural tamponade sealing the leak, the restriction of the CSF flow interfering with CSF absorption, and the change of dural resistance and stiffness [6].

The blind lumbar EBP procedure consists of the injection of 10 to $20 \mathrm{~cm}^{3}$ of autologous blood, [2] followed by putting the patient in the Trendelenburg position for 30 to 60 minutes, either supine, prone, or lateral, according to the location of the CSF leak. This will allow blood to travel over many spinal segments toward the site of the leak and will maximize the efficacy of this procedure [6]. The reported success rate is 36.8 to $89.5 \%$ after the first attempt [5, 9-11]. However, if necessary, a blind lumbar EBP can be repeated, with injection of 20 to $40 \mathrm{~cm}^{3}$ of blood, which may be beneficial in an additional 20 to $33 \%$ of patients [2]. Some authors recommend a minimum of 5 days between blood patches because local pain or radiculopathy may occur due to the high volume of blood injected [6]. 
When a repeated lumbar EBP is ineffective, a targeted EBP may be tried. It requires locating the spinal fluid leak and subsequent epidural injection of autologous blood at that level $[4,6,7,9]$. Although only few reports of targeted CTguided EBP are described in the literature, they appear to be more efficacious than blind lumbar patches [4], with relief of the symptoms after the first attempt in 71 to $87 \%$ of the cases reported $[5,9,12]$.

In most reported studies, a blind lumbar EBP was used in the treatment of SIH with thoracic or cervical leak sites because it is easier, and a targeted CT-guided EBP at thoracic or cervical levels was considered to be associated with a higher risk of procedural complications $[4,9,13]$. However, increasing reports show that the blind lumbar EBP is frequently unsuccessful in the treatment of cervical leaks, since an adequate volume of blood for patching the cervical leak is difficult to achieve with a lumbar injection. In some of these cases, a targeted EBP was required [1323]. A nonrandomized retrospective series evaluated the efficacy of a blind and targeted EBP after a single procedure in a population of $56 \mathrm{SIH}$ patients, and there was a significant difference in the results between the two methods. While the blind blood patch was successful in only $52 \%$ of the patients, the targeted EBP achieved a success rate of $87.1 \%$ [5].

The classical EBP is considered to be a safe procedure, and complications are usually mild and transient, such as blood leaks to the subcutaneous tissues, cauda equina syndrome, subdural hematoma, back and radicular pain, and epidural infection [24-26].

With current advances in neuroradiology, imagingguided procedures in the spine have been used more frequently because they appear to be more effective, safer, and more comfortable for the patient, especially when considering cervical leaks $[5,16]$. In the case of our patient, as the CSF leak was located at a cervical level, it was assumed that a blind lumbar EBP was less likely to be successful and a targeted CT-guided EBP was attempted with an excellent outcome and without complications.

Patients who fail to improve after conservative treatment and blood patching should be considered for a percutaneous fibrin glue injection or surgical intervention $[1,2,7]$.

Published data regarding the outcomes of patients with SIH is limited, but it is thought that most patients have good outcomes after combining conservative management and an EBP $[1,2,7,9]$.

In conclusion, SIH must be considered in the differential diagnosis of patients presenting with new daily persistent and orthostatic headache. In this report we present the targeted CT-guided EBP as an alternative in patients who are refractory to conservative treatment and the blind EBP. It seems to be a safe, minimally invasive, and highly effective treatment, which may avoid the need for a more aggressive approach, such as surgical intervention, in refractory patients.

\section{Consent}

Written informed consent was obtained.

\section{Conflict of Interests}

The authors declare that they have no conflict of interests.

\section{References}

[1] W. I. Schievink, "Spontaneous spinal cerebrospinal fluid leaks," Cephalalgia, vol. 28, no. 12, pp. 1347-1356, 2008.

[2] T. J. Schwedt and D. W. Dodick, "Spontaneous intracranial hypotension," Current Pain and Headache Reports, vol. 11, no. 1, pp. 56-61, 2007.

[3] J. R. Couch, "Spontaneous intracranial hypotension: the syndrome and its complications," Current Treatment Options in Neurology, vol. 10, no. 1, pp. 3-11, 2008.

[4] J. R. Couch and J. Persson, "Treatment of spontaneous intracranial hypotension with epidural blood patch: is a complex approach necessary or better than a simple one?" Acta Anaesthesiologica Scandinavica, vol. 56, no. 10, pp. 1207-1209, 2012.

[5] K.-I. Cho, H.-S. Moon, H.-J. Jeon, K. Park, and D.-S. Kong, "Spontaneous intracranial hypotension: efficacy of radiologic targeting vs blind blood patch," Neurology, vol. 76, no. 13, pp. 1139-1144, 2011.

[6] W. I. Schievink, "Spontaneous spinal cerebrospinal fluid leaks and intracranial hypotension," The Journal of the American Medical Association, vol. 295, no. 19, pp. 2286-2296, 2006.

[7] C. M. Wendl, F. Schambach, C. Zimmer, and A. Förschler, "CT myelography for the planning and guidance of targeted epidural blood patches in patients with persistent spinal CSF leakage," American Journal of Neuroradiology, vol. 33, no. 3, pp. 541-544, 2012.

[8] S. J. Chung, J. S. Kim, and M. C. Lee, "Syndrome of cerebral spinal fluid hypovolemia: clinical and imaging features and outcome," Neurology, vol. 55, no. 9, pp. 1321-1327, 2000.

[9] F. Amoozegar, D. Guglielmin, W. Hu, D. Chan, and W. J. Becker, "Spontaneous intracranial hypotension: recommendations for management," Canadian Journal of Neurological Sciences, vol. 40, no. 2, pp. 144-157, 2013.

[10] E. Ferrante, G. F. Rubino, S. Passarani, and I. Arpino, "Spontaneous intracranial hypotension," Journal of Neurosurgery, vol. 113, no. 2, pp. 397-398, 2010.

[11] S. J. Chung, J.-H. Lee, J.-H. Im, and M. C. Lee, "Short- and longterm outcomes of spontaneous CSF hypovolemia," European Neurology, vol. 54, no. 2, pp. 63-67, 2005.

[12] Y.-F. Wang, J.-F. Lirng, J.-L. Fuh, S.-S. Hseu, and S.-J. Wang, "Heavily T2-weighted MR myelography vs CT myelography in spontaneous intracranial hypotension," Neurology, vol. 73, no. 22, pp. 1892-1898, 2009.

[13] K. T. Sykes and X. Yi, "Intracranial hypotension headache caused by a massive cerebrospinal fluid leak successfully treated with a targeted C2 epidural blood patch: a case report," Pain Physician, vol. 16, no. 4, pp. 399-404, 2013.

[14] S. M. Hayek, M. Fattouh, T. Dews, L. Kapural, O. Malak, and N. Mekhail, "Successful treatment of spontaneous cerebrospinal fluid leak headache with fluoroscopically guided epidural blood patch: a report of four cases," Pain Medicine, vol. 4, no. 4, pp. 373-378, 2003.

[15] M. J. Cousins, D. Brazier, and R. Cook, "Intracranial hypotension caused by cervical cerebrospinal fluid leak: treatment with epidural blood patch," Anesthesia and Analgesia, vol. 98, no. 6, pp. 1794-1797, 2004. 
[16] A. Rai, C. Rosen, J. Carpenter, and V. Miele, "Epidural blood patch at C2: diagnosis and treatment of spontaneous intracranial hypotension," American Journal of Neuroradiology, vol. 26, no. 10, pp. 2663-2666, 2005.

[17] J. S. Donovan, C. W. Kerber, W. H. Donovan, and L. F. Marshall, "Development of spontaneous intracranial hypotension concurrent with grade IV mobilization of the cervical and thoracic spine: a case report," Archives of Physical Medicine and Rehabilitation, vol. 88, no. 11, pp. 1472-1473, 2007.

[18] P. W. H. Peng and R. Farb, "Spontaneous C1-2 CSF leak treated with high cervical epidural blood patch," Canadian Journal of Neurological Sciences, vol. 35, no. 1, pp. 102-105, 2008.

[19] A. Buvanendran, R. W. Byrne, M. Kari, and J. S. Kroin, "Occult cervical (C1-2) dural tear causing bilateral recurrent subdural hematomas and repaired with cervical epidural blood patch," Journal of Neurosurgery: Spine, vol. 9, no. 5, pp. 483-487, 2008.

[20] M. Allegri, F. Lombardi, V. M. Custodi et al., "Spontaneous cervical (C1-C2) cerebrospinal fluid leakage repaired with computed tomography- guided cervical epidural blood patch," Journal of Pain and Symptom Management, vol. 40, no. 3, pp. e9-e12, 2010.

[21] S. Y. Kim and J. H. Hong, "Epidural blood patches in a patient with multi-level cerebrospinal fluid leakage that was induced by spontaneous intracranial hypotension," The Korean Journal of Pain, vol. 23, no. 1, p. 46, 2010.

[22] P. Nipatcharoen and S. G. Tan, "High thoracic/cervical epidural blood patch for spontaneous cerebrospinal fluid leak: a new challenge for anesthesiologists," Anesthesia and Analgesia, vol. 113, no. 6, pp. 1476-1479, 2011.

[23] E. Wang and D. Wang, "Successful treatment of spontaneous intracranial hypotension due to prominent cervical cerebrospinal fluid leak with cervical epidural blood patch," Pain Medicine, 2015.

[24] P. Kalina, P. Craigo, and T. Weingarten, "Intrathecal injection of epidural blood patch: a case report and review of the literature," Emergency Radiology, vol. 11, no. 1, pp. 56-59, 2004.

[25] I. H. Tekkök, D. A. Carter, and R. Brinker, "Spinal subdural haematoma as a complication of immediate epidural blood patch," Canadian Journal of Anaesthesia, vol. 43, no. 3, pp. 306309, 1996.

[26] R. A. Beers, J. J. Cambareri, and G. S. Rodziewicz, "Acute deterioration of mental status following epidural blood patch," Anesthesia and Analgesia, vol. 76, no. 5, pp. 1147-1149, 1993. 


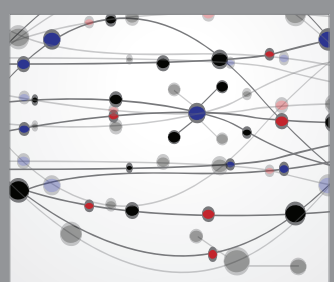

The Scientific World Journal
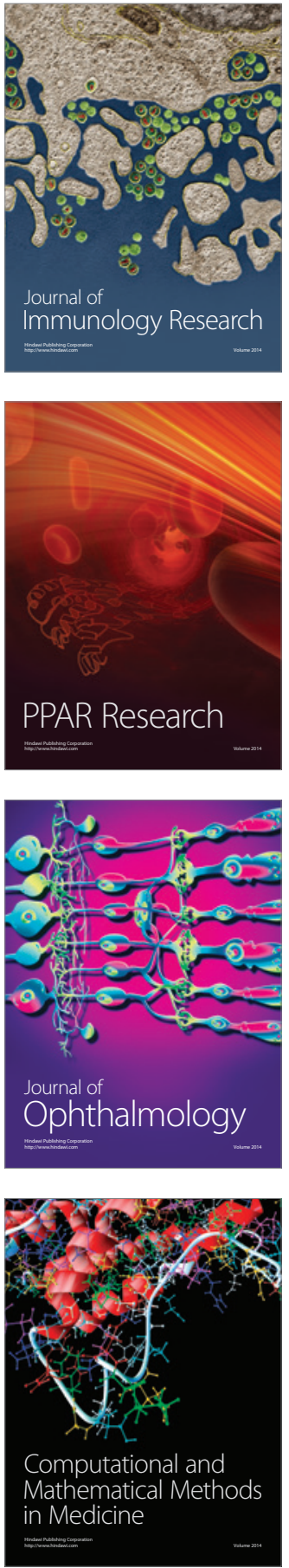

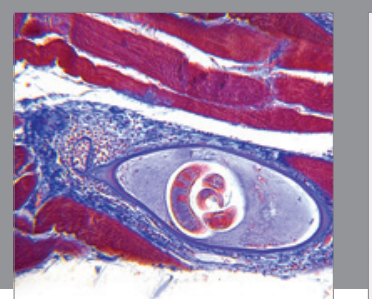

Gastroenterology Research and Practice

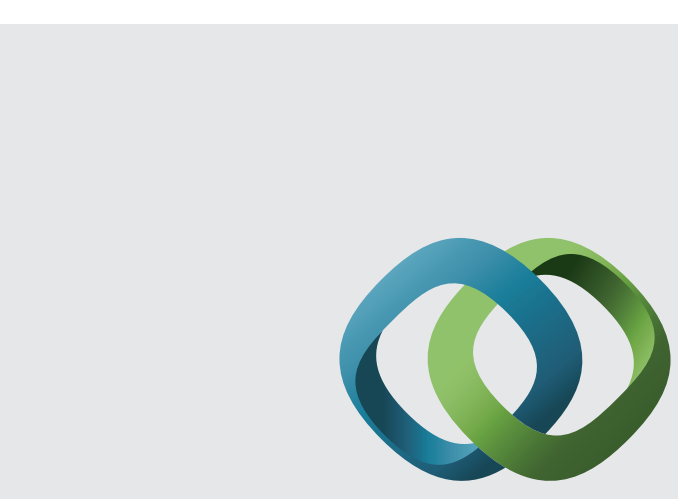

\section{Hindawi}

Submit your manuscripts at

http://www.hindawi.com
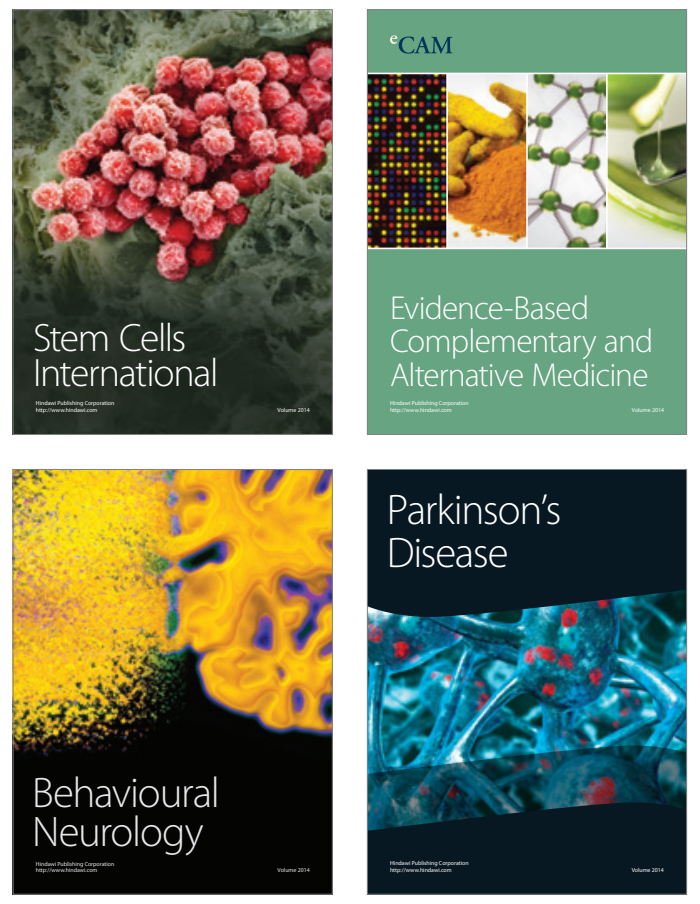
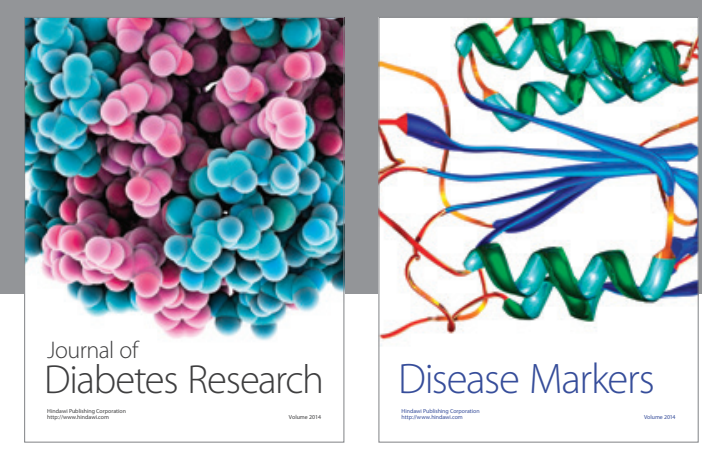

Disease Markers
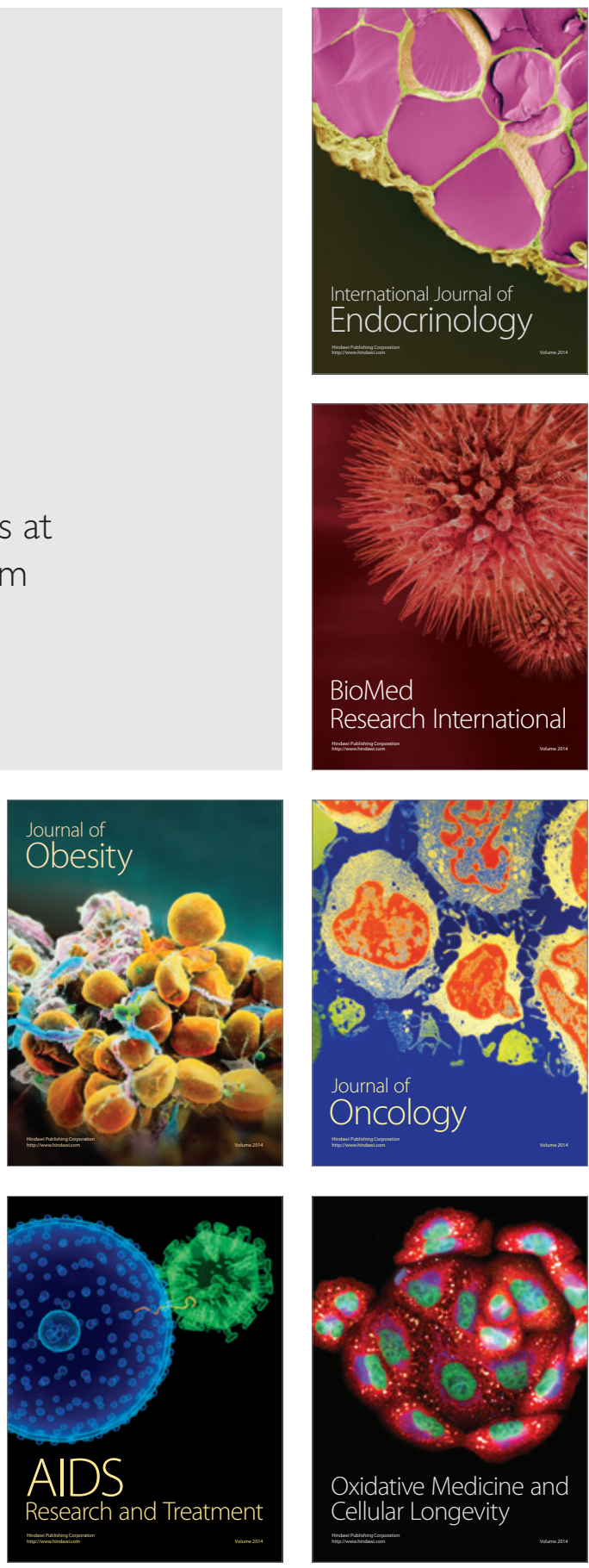